Article

\title{
Development of a Multiplex RT-qPCR for the Detection of Different Clades of Avian Influenza in Poultry
}

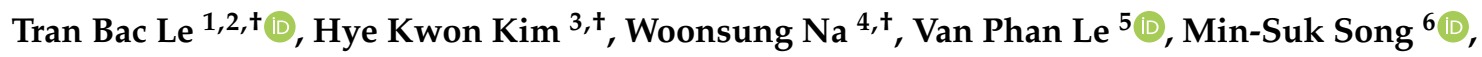 \\ Daesub Song ${ }^{7}$, Dae Gwin Jeong ${ }^{1,2, * \mathbb{D}}$ and Sun-Woo Yoon 1,2,* \\ 1 Infectious Diseases Research Center, Korea Research Institute of Bioscience and Biotechnology, \\ Daejeon 34141, Korea; letranbac86@kribb.re.kr \\ 2 Bio-Analytical Science Division, University of Science and Technology, Daejeon 34113, Korea \\ 3 Department of Microbiology, Chungbuk National University, Cheongju 28644, Korea; \\ khk1329@chungbuk.ac.kr \\ 4 College of Veterinary Medicine, Chonnam National University, Gwangju 61186, Korea; wsungna@gmail.com \\ 5 College of Veterinary Medicine, Vietnam National University of Agriculture, Hanoi 100000, Vietnam; \\ letranphan@gmail.com \\ 6 College of Medicine, Chungbuk National University, Cheongju 28644, Korea; songminsuk@chungbuk.ac.kr \\ 7 College of Pharmacy, Korea University, Sejong City 30019, Korea; songdaesop@gmail.com \\ * Correspondence: dgjeong@kribb.re.kr (D.G.J.); syoon@kribb.re.kr (S.-W.Y.); Tel.: +82-42-879-8411 (D.G.J.); \\ +82-42-879-8278 (S.-W.Y.) \\ + These authors contributed equally to this work.
}

Received: 6 December 2019; Accepted: 13 January 2020; Published: 15 January 2020

check for updates

\begin{abstract}
Since the initial detection of H5N1, a highly pathogenic avian influenza (HPAI) virus, in 1996 in China, numerous HPAI H5 lineages have been classified, and they continue to pose a threat to animal and human health. In this study, we developed a novel primer/probe set that can be employed to simultaneously detect pan-H5 HPAI and two clades, 2.3.2.1 and 2.3.4.4, of H5Nx viruses using reverse transcription quantitative polymerase chain reaction (RT-qPCR). The sensitivity and specificity of these primer sets and probes were confirmed with a number of different subtypes of influenza virus and the H5-HA gene plasmid DNA. In particular, the multiplex RT-qPCR assay was successfully applied to the simultaneous detection of H5 HPAI and different virus clades in clinical field samples from a poultry farm. Therefore, this multiplex assay and a novel detection primer set and probes will be useful for the laboratory diagnosis and epidemiological field studies of different circulating H5 HPAI virus clades in poultry and migratory wild birds.
\end{abstract}

Keywords: HPAI; H5Nx; Clade; simultaneous detection; field samples

\section{Introduction}

Influenza A virus (IAV), a member of the genus Orthomyxovirus, causes a highly contagious respiratory disease in avian species and mammals [1]. Except for influenza H17 and H18 viruses that originated from bats, the waterfowl are known as a primordial reservoir for all subtypes of IAV [1,2]. Unlike human influenza viruses, avian influenza (AI) viruses are classified into two pathotypes-low pathogenic avian influenza (LPAI) and HPAI-based on the pathogenicity in chickens and molecular determinants of the multibasic cleavage site motif in the hemagglutinin (HA) protein $[3,4]$. During the circulation of HPAI virus subtypes H5Nx and H7Nx in poultry, they proved to be highly pathogenic with mortality of up to $100 \%$. Sporadically, the H5N1 HPAI viruses cross the species barrier into 
humans. According to the WHO report [5], different subtypes of H5 HPAI viruses pose a substantial threat to global health with high mortality rate and huge economic burden to poultry farms.

Outbreaks of H5Nx HPAI viruses in poultry are increasing, and numerous clades of H5Nx HPAI have been classified since the first detection of the goose/Guangdong (Gs/GD) H5N1 HPAI virus in China [6]. However, the Gs/GD viruses and other IAVs have reassorted and diversified into 10 separate clades with more than 30 classified higher-order clades identified so far [7]. In particular, among the reassortment of novel H5Nx HPAI viruses, 2.3.2.1 and 2.3.4.4 clades of H5 HPAI viruses have become endemic in poultry populations in Asian countries, such as China, Vietnam, and South Korea [8,9]. The novel H5Nx viruses have reassorted with different NA subtypes, such as H5N2, $\mathrm{H} 5 \mathrm{~N} 5, \mathrm{H} 5 \mathrm{~N} 6$, and H5N8, in poultry and migratory birds [10]. The interaction between poultry and migratory wild birds and the complex overlapping flyways contribute to their long distance virus transmission [11]. The clade 2.3.2.1 of H5N1 HPAI virus was first identified in Hong Kong in 2004; since then other subclades, including 2.3.2.1a, 2.3.2.1b, and 2.3.2.1c, have evolved [12]. In addition, there was an outbreak of the $\mathrm{H} 5$ clade 2.3.4.4 virus around the East Asian-Australasian and American flyways in recent years [13-15]. Furthermore, these lineage viruses with different antigenic properties have been cocirculating. Thus, rapid and highly accurate methods are necessary for the detection of H5 HPAI viruses and clade identification.

Molecular diagnostic methods are used as the first step in the identification and control of the IAV outbreaks. Thus, a number of molecular methods, such as DNA microarrays [16], loop-mediated isothermal amplification tests [17], and restriction fragment mass analysis [18], have been developed and applied for rapid HA subtyping of the IAV field isolates [19]. Among these methods, RT-qPCR has been implemented in most reference diagnostics laboratories for the detection and characterization of IAVs. These diagnostic techniques were developed and used for subtyping or sequencing of novel viruses. However, due to the continuous circulation of H5Nx HPAI and other virus clades in avian species, it is very difficult to control viral transmission in poultry [3]. To effectively control and rapidly diagnose suspected infection on poultry farms, it is important to develop a rapid diagnostic assay for the simultaneous detection of different H5 HPAI viruses [20].

In this study, we tested our one-step multiplex method using clinical poultry samples to simultaneously detect pan-HPAI and two H5 clades. Thus, our one-step multiplex RT-qPCR assay can be used for the screening of virus-infected field samples.

\section{Materials and Methods}

\subsection{Reference Viruses and Field Samples Collection}

A total of 15 reference influenza virus samples were obtained from the Korea Research Institute of Bioscience and Biotechnology (KRIBB) and National Vietnam University of Agriculture. Coronavirus-229E and parainfluenza virus 1 were purchased from the Korea Bank for Pathogenic Viruses. Newcastle disease virus (VN1) and Infectious bronchitis virus (VNUA3) were kindly supported by Dr. Le Van Phan (National Vietnam University of Agriculture). All the reference influenza viruses were propagated in the allantoic fluid of 9-11 days old embryonated specific pathogen-free chicken eggs. All H5 HPAI experiments were conducted in a biosafety level 3 containment facility in KRIBB and biosafety level 2 plus facilities at the College of Veterinary Medicine, Vietnam National University of Agriculture, Hanoi, Vietnam. Field samples obtained from 12 farms in Vietnam were taken from domestic chickens with signs of respiratory illness in 2016 to 2018. For the screening of influenza positive field samples, all filed samples were analyzed using M-gene-specific RT-qPCR according to the WHO manual on animal influenza diagnosis and surveillance [21], and only M-gene positive field samples were used in the present study. 


\subsection{Primer and Probe Design}

To detect the pan-H5 HPAI-specific and HPAI clade-specific probes, H5Nx HA gene sequences of 1018 low pathogenic and 4301 highly pathogenic avian influenza viruses from the GenBank database of the National Center for Biotechnology Information or Influenza Research Database (IRD, www.fludb.org) were analyzed, and complementary sequences were designed using ClustalW in BioEdit software (7.2 version) to identify conserved regions. The probes were labeled with the following fluorescent dyes: FAM for clade 2.3.2.1 detection, HEX for clade 2.3.4.4 detection, or Texas Red for H5 HPAI detection. The following primers and probes were used: HA forward primer, CCAGCCAATGACCTYTGT; HA reverse primer, GRTAAGCCAYACCACATTTC; Pan H5-HPAI probe, Texas red-TGARGAAYTGAAACACCTATTGAGCAG-BHQ2; Clade 2.3.2.1 probe, FAM-TCCCTGGTATGAACATGCTGCGCT-BHQ1; Clade 2.3.4.4 probe, HEX-TACCCAGGGAVCCTCAATGA-BHQ1. All amplicons obtained using one set of HA primers were 201 base pairs in length.

\subsection{Sensitivity Analyses of One-Step Multiplex RT-qPCR Analysis}

To assess the reaction efficiency and limit of detection of our multiplex qPCR assay, viral H5-HA gene was synthesized using the full-length H5-HA gene sequence from A/environment/Korea/W150/2006 for HPAI detection, A/Environment/Korea/W541/2016 for clade 2.3.4.4 detection, or A/wild bird/Jiangsu/H184/2015 for clade 2.3.2.1 detection and cloned using the Mighty TA cloning kit according to the manufacturer's recommendations. After cloning, each H5-HA gene plasmid insertion was confirmed by DNA sequencing analysis. For the determination of limit of detection, one-step multiplex qPCR assays were performed using a serial 10 -fold dilution $\left(5 \times 10^{8}\right.$ to $5 \times 10^{0}$ copies of plasmid DNA/ $\mu \mathrm{L}$ ) of each plasmid; each qPCR assay was performed in triplicate. The plasmid copy number was calculated using the URI Genomics \& Sequencing Center program (http://cels.uri.edu/gsc/cndna.html).

\subsection{One-Step Multiplex RT-qPCR Analysis}

For the one-step multiplex RT-qPCR, viral RNA was extracted using the RNeasy Mini Kit (Hilden, Germany) following the manufacturer's instructions. The RT-qPCR assays were performed using the LightCycler 96 instrument and software (1.1 version). The one-step multiplex RT-qPCR reaction mixture contained $0.8 \mu \mathrm{L}$ of enzyme mixture, $4 \mu \mathrm{L}$ of $5 \times$ reaction buffer, $0.8 \mu \mathrm{L}$ of deoxynucleoside triphosphate (10 pmol) from a Qiagen (Hilden, Germany) one-step RT-qPCR kit, $1 \mu \mathrm{L}$ of each primer (10 pmol), $1 \mu \mathrm{L}$ of each probe $(5 \mu \mathrm{M}), 3 \mu \mathrm{L}$ RNA template and RNase-free water so that the final reaction volume reached $20 \mu \mathrm{L}$. The PCR thermocycling procedure was performed at $50{ }^{\circ} \mathrm{C}$ for $30 \mathrm{~min}$ and $94{ }^{\circ} \mathrm{C}$ for $10 \mathrm{~min}$ for the reverse transcription step; the next step was performed at $94{ }^{\circ} \mathrm{C}$ for $20 \mathrm{~s}$, $56{ }^{\circ} \mathrm{C}$ for $20 \mathrm{~s}$, and $72{ }^{\circ} \mathrm{C}$ for $30 \mathrm{~s}$ for 40 cycles. Fluorescence data were acquired during the annealing step to distinguish the positive and negative results. The RT-qPCR positive reaction of $c t$ value was set to 35 and RNase-free water was used as a negative control.

\subsection{Specificity Analyses of One-Step Multiplex RT-qPCR Analysis}

Specificity of our multiplex RT-qPCR system was evaluated with different influenza virus and other avian/human pathogens. total viral RNA samples from 1 human influenza virus (H1N1 subtype), 11 avian influenza viruses (H1N1, H3N3, H5N1, H5N2, H5N6, H5N8, H7N1, H9N2, and H10N1 subtype), 1 equine influenza virus (H3N8 subtype), 1 canine influenza virus (H3N2), 1 swine influenza virus (H3N2 subtype), avian infectious bronchitis virus, Newcastle disease virus, human Coronavirus-229E, and human Parainfluenza were assessed using one-step multiplex RT-qPCR described in this study. 


\subsection{H5 HPAI Clade Assignment of Filed Samples}

All H5 HPAI-positive field samples were identified by conventional reverse transcription polymerase chain reaction (RT-PCR), and the HA gene was amplified using the following primer set: forward 5'-GCATTGGYTAYCATGCAAAYA-3' and reverse 5'-TTGCTRTGGTGRTAYCCATACA-3'. Specific amplicons were purified from 1.5\% agarose gels using a QIAquick Gel Extraction Kit (Hilden, Germany) and sequenced using an ABI PRISM 3730 DNA Sequencer. The sequences were compiled and edited using the BioEdit program (www.mbio.ncsu.edu/BioEdit/bioedit.html). Based on their sequences, all H5 HPAI-positive field viruses were classified as the H5 HPAI clade using Highly Pathogenic H5N1 Clade Classification Tool in the Influenza Research Database.

\section{Results}

\subsection{Sensitivity of One-Step Multiplex RT-qPCR Assay}

To develop the one-step multiplex RT-qPCR assay, a specific primer set and the probes for pan-H5 HPAI and two clades, 2.3.4.4 or 2.3.2.1, were designed. In order to enhance the simultaneous detection specificity of a target gene, the H5 HPAI HA gene sequences from different years and various clades of H5 HPAI strains were downloaded from the GenBank database; primers and probes for the most conserved regions of the HA gene (Figure 1) were designed. To optimize our one-step multiplex qPCR, three HA gene plasmids, containing the H5 HPAI HA genes of two clades, were amplified and used as a quantitative standard in our system. Each plasmid was serially diluted $\left(5 \times 10^{8}\right.$ to $5 \times 10^{0}$ copies per microliter), and the sensitivity of our primer/probe set was determined using the singleplex and multiplex qPCR assays targeting pan-H5 HPAI, clade 2.3.4.4, or 2.3.2.1. The standard curves, generated for these targets under both qPCR conditions, showed linearity $\left(R^{2}=0.9918\right.$ to 0.9997 , Figure 2). Also, we showed that the detection limit under both $\mathrm{PPCR}$ conditions was $5 \times 10^{1}$ copies per microliter (Table 1). The cut-off for the cycle threshold of RT-qPCR was set to 35.

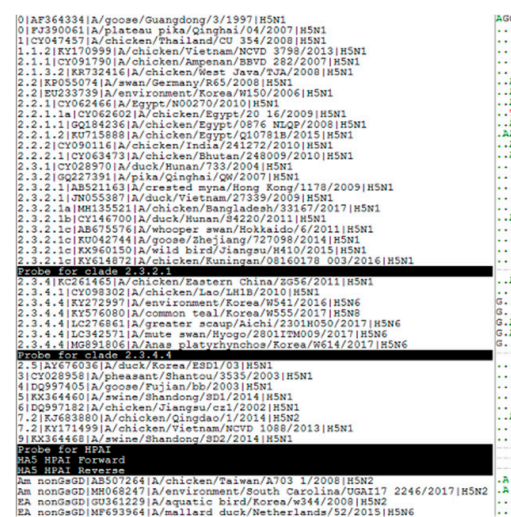

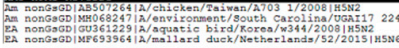

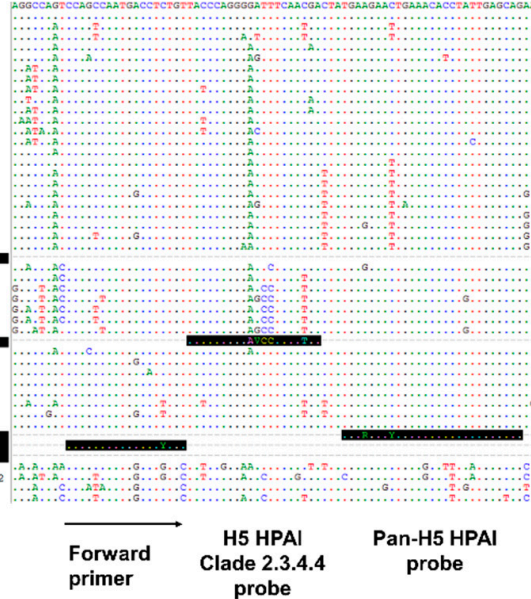

probe

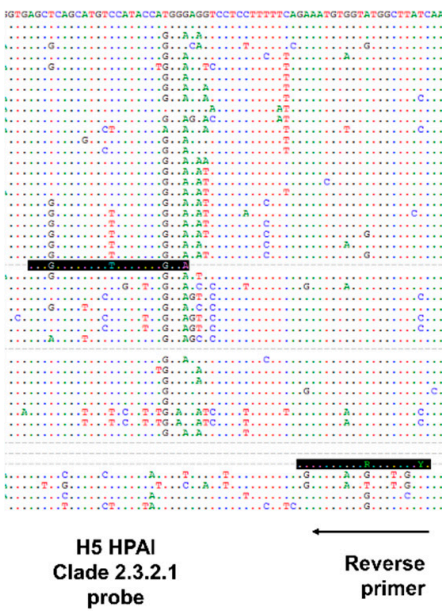

Figure 1. Design of the primer/probe set for the one-step multiplex RT-qPCR assay. Alignment of nucleotide sequences within the hemagglutinin (HA) gene of $\mathrm{H} 5 \mathrm{Nx}$ avian influenza viruses that are representative of highly pathogenic avian influenza (HPAI) clades and low pathogenic avian influenza (LPAI) virus. The sequences of primers and probes specific to each target are on black background, and the reverse primer and probe specific for clade 2.3.2.1 show reverse complement sequence $(R=A / G$, $\mathrm{Y}=\mathrm{C} / \mathrm{T}, \mathrm{V}=\mathrm{A} / \mathrm{C} / \mathrm{G})$. 
A) Singleplex realtime-qPCR of H5 HPAl detection

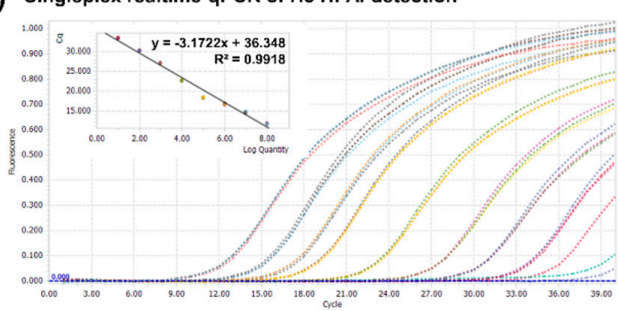

C) Singleplex realtime-qPCR of clade 2.3.2.1 detection

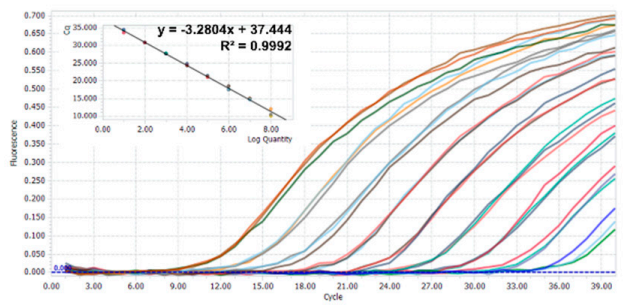

E) Singleplex realtime-qPCR of clade 2.3.4.4 detection

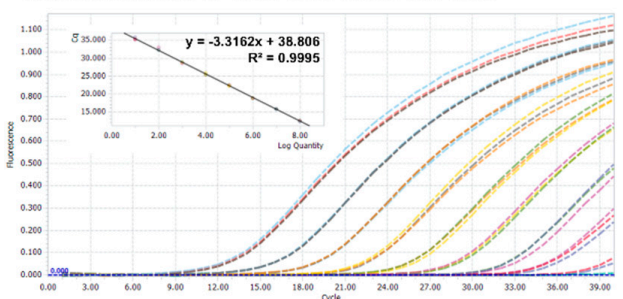

B) Multiplex realtime-qPCR of H5 HPAl detection

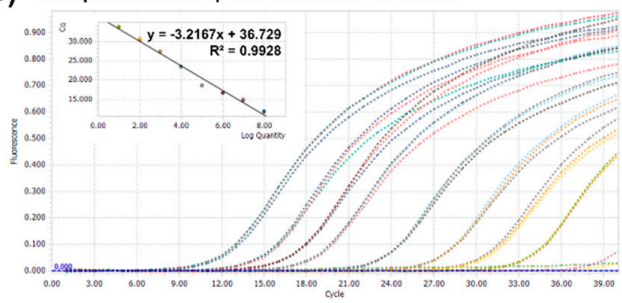

D) Multiplex realtime-qPCR of clade 2.3.2.1 detection

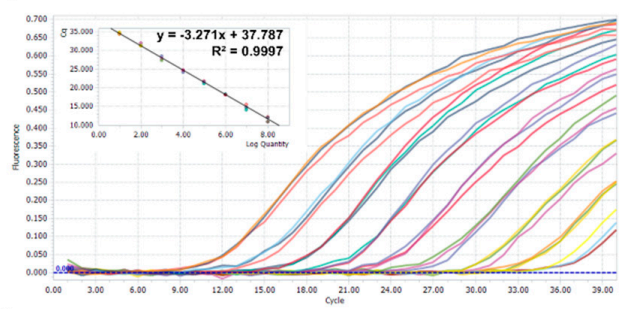

F) Multiplex realtime-qPCR of clade 2.3.4.4 detection

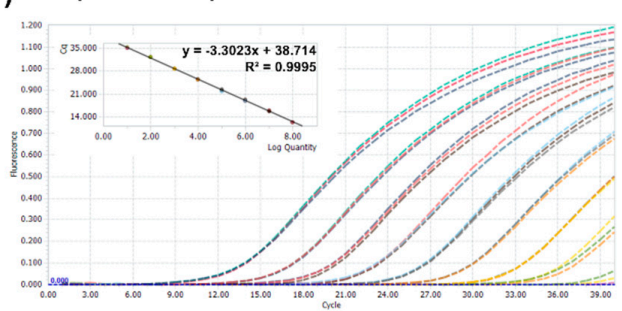

Figure 2. Amplification plots and standard curves of the singleplex and multiplex realtime-qPCR assays using target-specific plasmid DNA. Comparison of the singleplex $(\mathbf{A}, \mathbf{C}, \mathbf{E})$ and multiplex $(\mathbf{B}, \mathbf{D}, \mathbf{F})$ realtime-qPCR assays was performed using standard plasmids corresponding to H5 HPAI and clades 2.3.4.4 and 2.3.2.1; each plasmid solution was serially diluted down to $5 \times 10^{8}$ to $5 \times 10^{0}$ copies of plasmid DNA/microliter. The cycle threshold in amplification plots, correlation coefficient (R2) and slope of the standard curve for the assays were drawn automatically by Lightcycler 96 software. Each concentration had triple replicates.

Table 1. Sensitivity of the single and multiplex assays.

\begin{tabular}{|c|c|c|c|c|c|c|c|}
\hline \multirow[b]{2}{*}{ Specificity } & \multirow[b]{2}{*}{ Fluorescence Dye } & \multicolumn{3}{|c|}{ Singleplex Assay } & \multicolumn{3}{|c|}{ Multiplex Assay } \\
\hline & & $\begin{array}{c}\text { Limit of } \\
\text { Detection }\end{array}$ & $\begin{array}{l}\text { Mean } c t \\
\text { Value }\end{array}$ & SD & $\begin{array}{c}\text { Limit of } \\
\text { Detection }\end{array}$ & $\begin{array}{l}\text { Mean } c t \\
\text { Value }\end{array}$ & SD \\
\hline Clade 2.3.4.4 & Hex & $5 \times 10^{1}$ & 34.39 & 0.025 & $5 \times 10^{1}$ & 33.84 & 0.03 \\
\hline Clade2.3.2.1 & FAM & $5 \times 10^{1}$ & 33.83 & 0.020 & $5 \times 10^{1}$ & 34.5 & 0.025 \\
\hline Pan-H5 HPAI & Texas red & $5 \times 10^{1}$ & 34.12 & 0.061 & $5 \times 10^{1}$ & 33.89 & 0.06 \\
\hline
\end{tabular}

Each concentration had triple replicates and the mean and SD were conducted using GraphPad Prism software (version 5.1).

\subsection{Specificity of One-Step Multiplex RT-qPCR Assay}

To assess analytical specificity of the one-step multiplex RT-qPCR assay, strains of different subtypes or clades of influenza A virus, as well as other avian or human origin viruses, infectious bronchitis virus, Newcastle disease virus, coronavirus-229E strain, and parainfluenza virus 1, were tested. As we expected, all H5 HPAI and two clade viruses were successfully detected, while the other influenza A virus subtypes and viruses of other origin were not amplified by our multiplex RT-qPCR (Table 2). In addition, the H5 LPAI subtype viruses were not detected using our specific H5 HPAI primer/probe set. Interestingly, although the H5N1 HPAI subtypes of A/environment/Korea/W150/2006 (clade 2.2) and A/duck/Vietnam/NCVD-1648/2012 (clade 2.3.2.1) are the same, our primer/probe set detected only clade 2.3.2.1. These results show that our multiplex RT-qPCR assay is highly specific and exhibits no cross-reactivity for H5 HPAI and the other clades. 
Table 2. Reference virus strains used for specificity analysis of the developed RT-qPCR.

\begin{tabular}{|c|c|c|c|c|c|c|c|}
\hline \multirow{2}{*}{ Virus Name } & \multirow{2}{*}{ Host } & \multirow{2}{*}{ Accession Number } & \multirow{2}{*}{ Subtype } & \multicolumn{3}{|c|}{ Mean $c t$ Value } & \multirow{2}{*}{ Comments } \\
\hline & & & & H5 HPAI & 2.3.2.1c Clade & 2.3.4.4 Clade & \\
\hline A/California/04/2009 & Human & GQ280797 & H1N1 & ND & ND & ND & \\
\hline A/wild bird/Korea/SK14/2014 & Avian & KX066871 & $\mathrm{H} 1 \mathrm{~N} 1$ & ND & ND & ND & LPAI \\
\hline A/swine/Korea/CAN04/2005 & Swine & EU798790 & H3N2 & ND & ND & ND & \\
\hline $\mathrm{A} /$ canine/Korea/01/2007 & Canine & JX163256 & H3N2 & ND & ND & ND & \\
\hline A/aquatic bird/SouthKorea/sw006/2016 & Avian & MG386182 & H3N3 & ND & ND & ND & LPAI \\
\hline A/equine/Kyonggi/SA1/2011 & Equine & JX844146 & H3N8 & ND & ND & ND & \\
\hline A/environment/Korea/W150/2006 & Avian & EU233739 & H5N1 & 21.55 & ND & ND & Clade 2.2 \\
\hline A/duck/Vietnam/NCVD-1648/2012 & Avian & KY171342 & H5N1 & 20.58 & 21.6 & ND & Clade 2.3.2.1c \\
\hline A/aquatic bird/ South Korea/sw007/2015 & Avian & MG386197 & H5N3 & ND & ND & ND & LPAI \\
\hline $\mathrm{A} /$ Environment/Korea/W541/2016 & Avian & KY272997 & H5N6 & 22.13 & ND & 21.36 & Clade 2.3.4.4 \\
\hline A/Common Teal/Korea/W555/2017 & Avian & KY576080 & $\mathrm{H} 5 \mathrm{~N} 8$ & 20.82 & ND & 19.02 & Clade 2.3.4.4 \\
\hline A/aquatic bird/ South Korea/sw001/2015 & Avian & MF987893 & H7N1 & ND & ND & ND & LPAI \\
\hline A/Chicken/Korea/MS96/1996 & Avian & GU053186 & H9N2 & ND & ND & ND & LPAI \\
\hline A/Aquatic bird/South Korea/SW1/2018 & Avian & MK539837 & H10N1 & ND & ND & ND & LPAI \\
\hline Infectious bronchitis virus & Avian & KY992863 & VNUA3 & ND & ND & ND & \\
\hline Newcastle disease virus & Avian & КС607878 & VN1 & ND & ND & ND & \\
\hline Coronavirus-229E, strain & Human & AY386391 & KUMC-9 & ND & ND & ND & \\
\hline Parainfluenza virus 1 & Human & MG255129 & KUMC-44 & ND & ND & ND & \\
\hline
\end{tabular}

ND: not detected. Each concentration had triple replicates. 


\subsection{Application of One-Step Multiplex RT-qPCR Assay Using Clinical Samples}

To detect H5 HPAI in fecal samples using our one-step multiplex RT-qPCR assay, we obtained a total of 4350 fecal samples from domestic chickens with signs of respiratory illness in Vietnam, and viral RNA was isolated from these samples. A total of 68 samples were confirmed influenza positive by M-gene-specific RT-qPCR. Among the IAV-positive clinical samples, 51 samples were H5 HPAI-positive, and each clade was successfully analyzed using our one-step multiplex RT-qPCR assay (Table 3 and Figure 3). Next, we confirmed the sequence of all H5 HPAI-positive samples using the universal HA sequencing primers, forward 5'-GCATTGGYTAYCATGCAAAYA-3' and reverse $5^{\prime}$-TTGCTRTGGTGRTAYCCATACA-3' , and classified each clade using the Influenza Research Database. Interestingly, when we have compared with sequencing analysis and clade identification using each amplicon, the primers and probes for the pan-H5 HPAI-specific and two clade-specific multiplex RT-qPCR assays were well-matched (Figure 3D). These results show that our one-step multiplex RT-qPCR assay could be a promising technique for specific discrimination of multiple H5 HPAI viruses in field samples from infected avian species.

A)
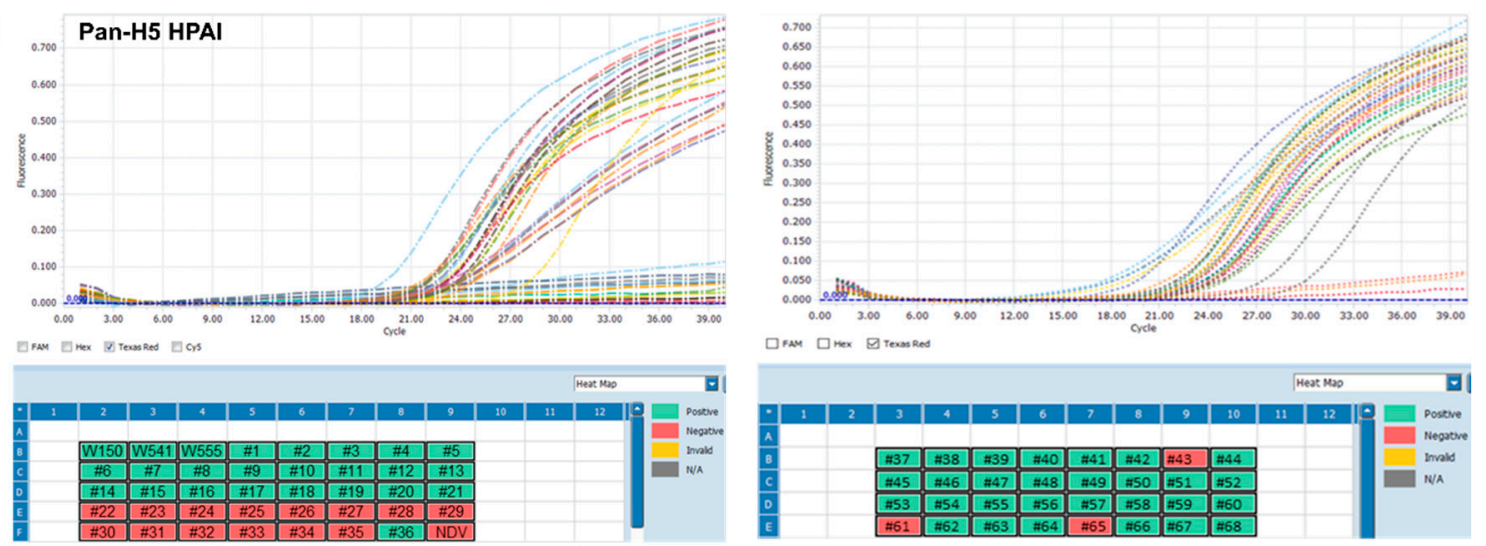

B) $\quad$ H5 HPAl/Clade 2.3.2.1
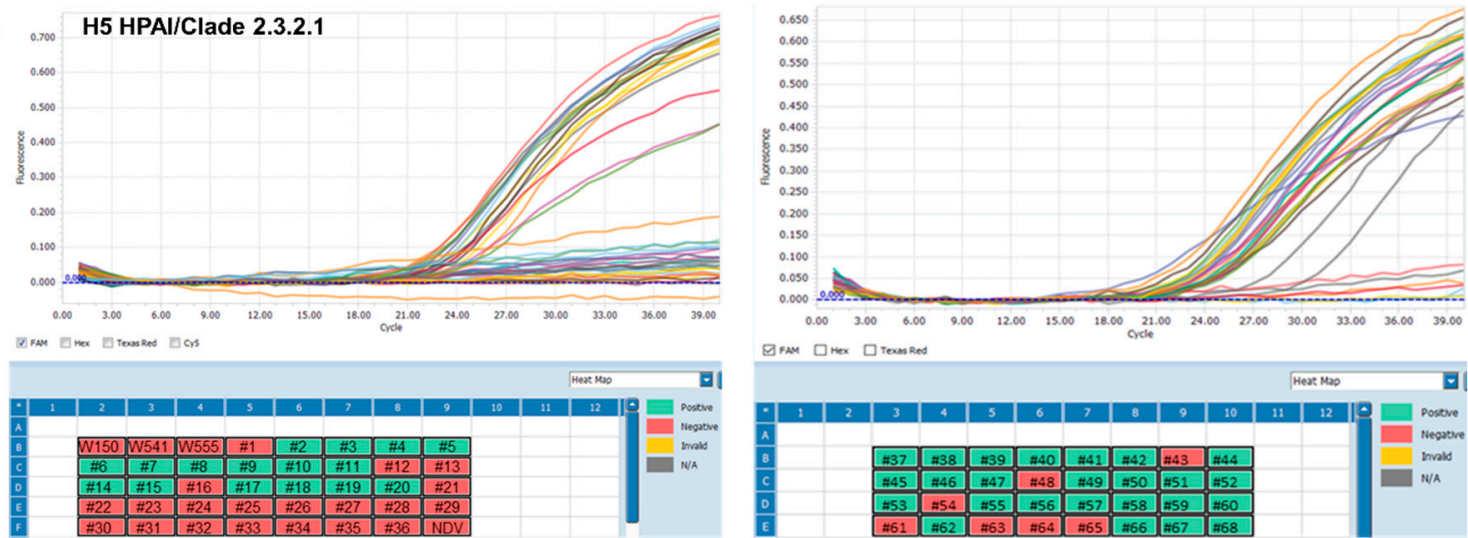

Figure 3. Cont. 
C)
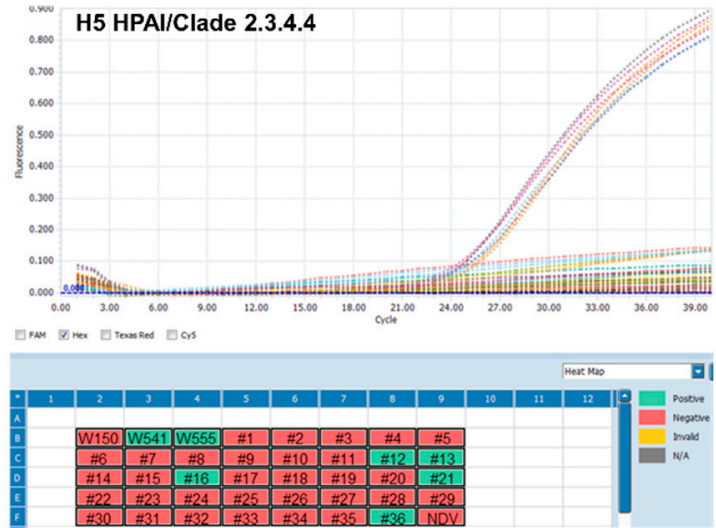

D)

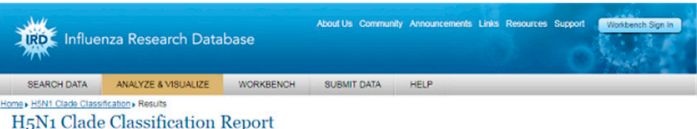

${ }_{5} \mathrm{~N}_{1}$ Clade Classification Repo

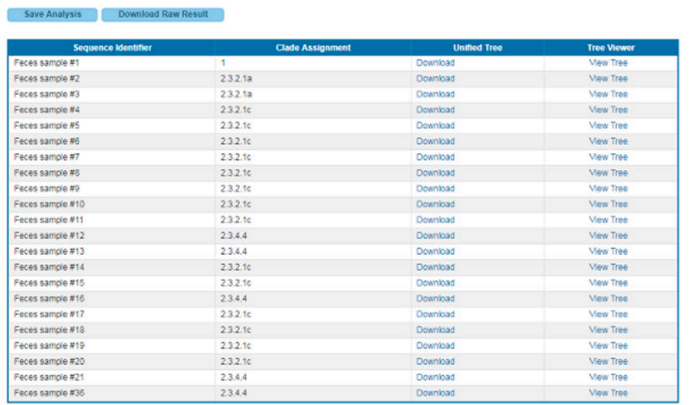

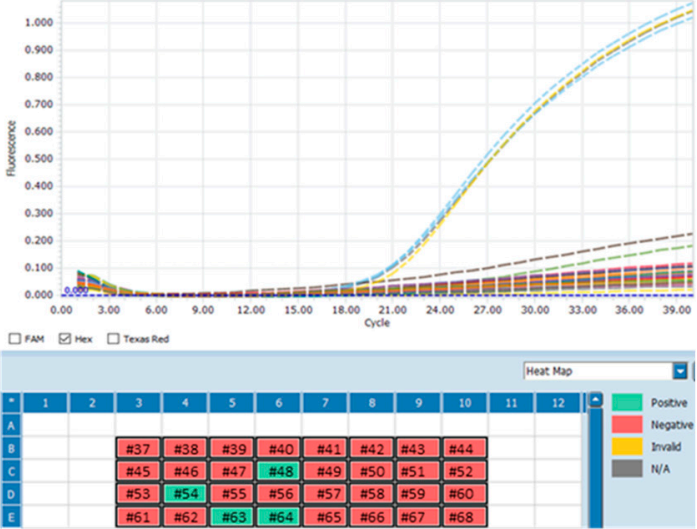

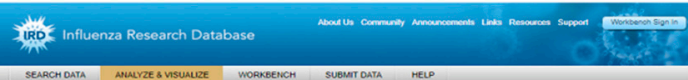

$\mathrm{H}_{5} \mathrm{~N}_{1}$ Clade Classification Report

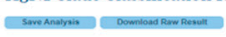

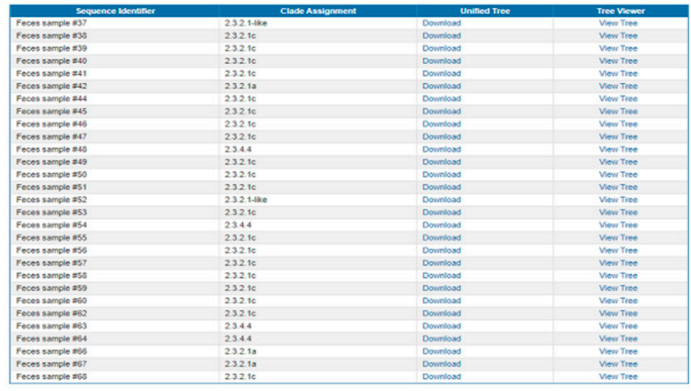

Figure 3. The one-step multiplex RT-qPCR assay for the detection of pan-H5 HPAI (A), H5 HPAI 2.3.2.1 clade (B), and H5 HPAI 2.3.4.4 clade (C) in field-infected feces samples. The validity of this assay was confirmed using M-gene positive field samples, H5Nx HPAI viruses for positive control, and Newcastle Disease virus for negative control. The results were visualized using an intuitive heat-map (green color-positive result, red color-negative result). Each concentration had triple replicates. (D) Clade classification of HPAI H5 subtype viruses from field-infected feces samples. The HA1 partial sequences of the HA gene with multiple basic cleavage sites were obtained by conventional RT-PCR and all H5 HPAI-positive sequences were verified using the Influenza Research Database (www.fludb.org). 
Table 3. Application of the multiplex RT-qPCR to feces samples from field-infected chickens.

\begin{tabular}{|c|c|c|c|c|c|c|c|c|}
\hline \multirow{3}{*}{ Samples Description } & \multicolumn{7}{|c|}{ ct Value } & \multirow{3}{*}{ Clade ** } \\
\hline & \multirow{2}{*}{$\mathrm{IAV}^{*}$} & \multicolumn{2}{|c|}{ HPAI } & \multicolumn{2}{|c|}{ 2.3.2.1 } & \multicolumn{2}{|c|}{ 2.3.4.4 } & \\
\hline & & Singleplex & Multiplex & Singleplex & Multiplex & Singleplex & Multiplex & \\
\hline A/environment/Korea/W150/2006 & + & 20.32 & 20.04 & ND & ND & ND & ND & 2.2 \\
\hline A/Environment/Korea/W541/2016 & + & 20.68 & 21.33 & ND & ND & 21.32 & 22.09 & 2.3.4.4 \\
\hline A/Common Teal/Korea/W555/2017 & + & 19.79 & 21.81 & ND & ND & 21.62 & 22.01 & 2.3.4.4 \\
\hline Feces sample \#1 & + & 25.35 & 26.3 & ND & ND & ND & $\mathrm{ND}$ & 1 \\
\hline Feces sample \#2 & + & 21.82 & 21.29 & 23.72 & 24.07 & ND & ND & 2.3.2.1a \\
\hline Feces sample \#3 & + & 19.5 & 19.32 & 20.97 & 22.12 & ND & ND & 2.3.2.1a \\
\hline Feces sample \#4 & + & 19.84 & 19.72 & 20.05 & 20.93 & ND & ND & 2.3.2.1c \\
\hline Feces sample \#5 & + & 19.99 & 19.2 & 19.89 & 21.32 & ND & ND & 2.3.2.1c \\
\hline Feces sample \#6 & + & 21.37 & 21.53 & 22.18 & 21.9 & ND & ND & 2.3.2.1c \\
\hline Feces sample \#7 & + & 20.24 & 20.35 & 21.07 & 20.75 & ND & ND & 2.3.2.1c \\
\hline Feces sample \#8 & + & 19.88 & 19.57 & 19.93 & 20.49 & ND & ND & 2.3.2.1c \\
\hline Feces sample \#9 & + & 23.13 & 22.63 & 23.19 & 23.56 & ND & ND & 2.3.2.1c \\
\hline Feces sample \#10 & + & 22.29 & 22.15 & 22.64 & 23.26 & ND & ND & 2.3.2.1c \\
\hline Feces sample \#11 & + & 20.33 & 19.68 & 20.4 & 21.1 & ND & ND & 2.3.2.1c \\
\hline Feces sample \#12 & + & 20.13 & 21 & ND & ND & 20.7 & 21.97 & 2.3.4.4 \\
\hline Feces sample \#13 & + & 19.48 & 22.59 & ND & ND & 22.33 & 21.24 & 2.3.4.4 \\
\hline Feces sample \#14 & + & 20.2 & 19.97 & 20.54 & 20.45 & ND & ND & 2.3.2.1c \\
\hline Feces sample \#15 & + & 22.05 & 21.58 & 21.93 & 22.31 & ND & ND & 2.3.2.1c \\
\hline Feces sample \#16 & + & 21.64 & 23.66 & ND & ND & 23.54 & 23.45 & 2.3.4.4 \\
\hline Feces sample \#17 & + & 20.85 & 20.56 & 20.88 & 21.44 & ND & ND & 2.3.2.1c \\
\hline Feces sample \#18 & + & 19.49 & 19.65 & 20.15 & 20.78 & ND & ND & 2.3.2.1c \\
\hline Feces sample \#19 & + & 20.92 & 21.02 & 21.6 & 21.98 & ND & ND & 2.3.2.1c \\
\hline Feces sample \#20 & + & 20.68 & 20.31 & 20.87 & 21.26 & ND & ND & 2.3.2.1c \\
\hline Feces sample \#21 & + & 22.04 & 23.54 & ND & ND & 23.15 & 23.13 & 2.3.4.4 \\
\hline Feces sample \#22 & + & ND & ND & ND & ND & ND & ND & LPAI \\
\hline
\end{tabular}


Table 3. Cont

\begin{tabular}{|c|c|c|c|c|c|c|c|c|}
\hline \multirow{3}{*}{ Samples Description } & \multicolumn{7}{|c|}{ ct Value } & \multirow{3}{*}{ Clade ** } \\
\hline & \multirow{2}{*}{$\mathrm{IAV}^{*}$} & \multicolumn{2}{|c|}{ HPAI } & \multicolumn{2}{|c|}{ 2.3.2.1 } & \multicolumn{2}{|c|}{ 2.3.4.4 } & \\
\hline & & Singleplex & Multiplex & Singleplex & Multiplex & Singleplex & Multiplex & \\
\hline Feces sample \#23 & + & ND & ND & $\mathrm{ND}$ & ND & ND & ND & LPAI \\
\hline Feces sample \#24 & + & ND & ND & ND & ND & ND & ND & LPAI \\
\hline Feces sample \#25 & + & ND & ND & ND & ND & ND & ND & LPAI \\
\hline Feces sample \#26 & + & ND & ND & ND & ND & ND & ND & LPAI \\
\hline Feces sample \#27 & + & ND & ND & ND & ND & ND & ND & LPAI \\
\hline Feces sample \#28 & + & ND & ND & ND & ND & ND & ND & LPAI \\
\hline Feces sample \#29 & + & ND & ND & ND & ND & ND & ND & LPAI \\
\hline Feces sample \#30 & + & ND & ND & ND & ND & ND & ND & LPAI \\
\hline Feces sample \#31 & + & ND & ND & ND & ND & ND & ND & LPAI \\
\hline Feces sample \#32 & + & ND & ND & ND & ND & ND & ND & LPAI \\
\hline Feces sample \#33 & + & ND & ND & ND & ND & ND & ND & LPAI \\
\hline Feces sample \#34 & + & ND & ND & ND & ND & ND & ND & LPAI \\
\hline Feces sample \#35 & + & ND & ND & ND & ND & ND & ND & LPAI \\
\hline Feces sample \#36 & + & 23.21 & 24.33 & ND & ND & 23.86 & 23.92 & 2.3.4.4 \\
\hline Feces sample \#37 & + & 26.71 & 27.4 & 27.01 & 27.48 & ND & ND & 2.3.2.1 \\
\hline Feces sample \#38 & + & 20.49 & 21.48 & 21.01 & 20.98 & ND & ND & 2.3.2.1c \\
\hline Feces sample \#39 & + & 22.27 & 22.93 & 22.65 & 23.11 & ND & ND & 2.3.2.1c \\
\hline Feces sample \#40 & + & 21.89 & 22.36 & 21.82 & 22.56 & ND & ND & 2.3.2.1c \\
\hline Feces sample \#41 & + & 22.92 & 23.31 & 22.91 & 23.37 & ND & ND & 2.3.2.1c \\
\hline Feces sample \#42 & + & 19.46 & 19.62 & 19.38 & 19.96 & ND & ND & 2.3.2.1a \\
\hline Feces sample \#43 & + & ND & ND & ND & ND & ND & ND & LPAI \\
\hline Feces sample \#44 & + & 23.17 & 23.71 & 23.16 & 24.16 & ND & ND & 2.3.2.1c \\
\hline Feces sample \#45 & + & 21.62 & 22.37 & 21.88 & 22.75 & ND & ND & 2.3.2.1c \\
\hline Feces sample \#46 & + & 29.58 & 29.98 & 29.41 & 30.42 & ND & ND & 2.3.2.1c \\
\hline Feces sample \#47 & + & 23.36 & 23.21 & 23.86 & 23.76 & ND & ND & 2.3.2.1c \\
\hline Feces sample \#48 & + & 18.88 & 18.94 & ND & ND & 19.88 & 20.05 & 2.3 .4 .4 \\
\hline
\end{tabular}


Table 3. Cont

\begin{tabular}{|c|c|c|c|c|c|c|c|c|}
\hline \multirow{3}{*}{ Samples Description } & \multicolumn{7}{|c|}{ ct Value } & \multirow{3}{*}{ Clade *: } \\
\hline & \multirow{2}{*}{ IAV * } & \multicolumn{2}{|c|}{ HPAI } & \multicolumn{2}{|c|}{ 2.3.2.1 } & \multicolumn{2}{|c|}{ 2.3.4.4 } & \\
\hline & & Singleplex & Multiplex & Singleplex & Multiplex & Singleplex & Multiplex & \\
\hline Feces sample \#49 & + & 23.6 & 24.3 & 23.42 & 24.52 & ND & ND & 2.3.2.1c \\
\hline Feces sample \#50 & + & 23.9 & 24.14 & 24.16 & 24.6 & ND & ND & 2.3.2.1c \\
\hline Feces sample \#51 & + & 22.32 & 22.85 & 22.71 & 23.22 & ND & ND & 2.3.2.1c \\
\hline Feces sample \#52 & + & 24.67 & 24.4 & 24.36 & 24.37 & ND & ND & 2.3.2.1c \\
\hline Feces sample \#53 & + & 21.32 & 22.06 & 21.22 & 21.71 & ND & ND & 2.3.2.1c \\
\hline Feces sample \#54 & + & 18.97 & 18.53 & ND & ND & 18.3 & 18.94 & 2.3 .4 .4 \\
\hline Feces sample \#55 & + & 23.3 & 23.22 & 23.17 & 22.93 & ND & ND & 2.3.2.1c \\
\hline Feces sample \#56 & + & 22.42 & 22.79 & 22.94 & 23 & ND & ND & 2.3.2.1c \\
\hline Feces sample \#57 & + & 22.8 & 22.92 & 22.43 & 22.36 & ND & ND & 2.3.2.1c \\
\hline Feces sample \#58 & + & 25.22 & 25.04 & 24.17 & 25.05 & ND & ND & 2.3.2.1c \\
\hline Feces sample \#59 & + & 23.57 & 23.83 & 23.91 & 23.38 & ND & ND & 2.3.2.1c \\
\hline Feces sample \#60 & + & 23.06 & 23.78 & 23.54 & 23.39 & ND & ND & 2.3.2.1c \\
\hline Feces sample \#61 & + & ND & ND & ND & ND & ND & ND & LPAI \\
\hline Feces sample \#62 & + & 24.81 & 24.75 & 23.93 & 24.55 & ND & ND & 2.3.2.1c \\
\hline Feces sample \#63 & + & 18.6 & 18.09 & ND & ND & 18.93 & 19.16 & 2.3.4.4 \\
\hline Feces sample \#64 & + & 18.16 & 18.2 & ND & ND & 19.16 & 19.3 & 2.3.4.4 \\
\hline Feces sample \#65 & + & ND & ND & ND & ND & ND & ND & LPAI \\
\hline Feces sample \#66 & + & 23.79 & 23.94 & 24.28 & 24.4 & ND & ND & 2.3.2.1a \\
\hline Feces sample \#67 & + & 23.78 & 23.78 & 23.83 & 24.25 & ND & ND & 2.3.2.1a \\
\hline Feces sample \#68 & + & 24.25 & 24.37 & 24.47 & 24.66 & ND & ND & 2.3.2.1c \\
\hline
\end{tabular}

ND: not detected; LPAI: low-pathogenic avian influenza. * Primers and probes set for M gene specific performed using the protocol from the WHO manual on animal influenza diagnosis and surveillance [21]. + Influenza positive sample. ${ }^{* *}$ All sequences were confirmed from GenBank at the National Center for Biotechnology Information (NCBI) or the Influenza Research Database (IRD, www.fludb.org). Each concentration had triple replicates. 


\section{Discussion}

Since H5N1 HPAI viruses was first identified in 1996, diverse subtypes of H5 HPAI were classified. Global and domestic spread of these virus caused huge losses to the poultry industry [18]. Novel H5Nx HPAI viruses appeared due to reassortment of the HA gene with different NA genes. According to the WHO report [22], H5Nx HPAI viruses have been classified into 13 clades based on their HA sequences. Recently, new clade classifications appeared (1.1.1, 7.1, 2.1.3.1, 2.1.3.3, 2.2.1.1, 2.2.1.1a, 2.2.2, 2.2.2.1, 2.3.2.1, 2.3.4.1, 2.3.4.3, and 2.3.4.4) owing to the emerging HPAI virus subtypes H5N2, H5N3, H5N5, H5N6, and H5N8 [18]. Among several new clades, two clades of H5 HPAI virus, 2.3.4.4 and 2.3.2.1, have been recently prevalent and dominant in Asia such as China [23], South Korea [24], and Vietnam [25]. In particular, 2.3.4.4 clade of $\mathrm{H} 5$ virus has spread to China and neighboring countries, such as South Korea [24], Vietnam [25], and Laos [26] between 2013 and 2016.

Despite many efforts to control the spread of H5 HPAI viruses [27] in poultry, such as development of a vaccine and diagnostic systems [28], viruses evolved and infected wild birds and domestic poultry. In particular, to effectively monitor and control H5Nx HPAI virus transmission, a number of diagnostic techniques, including virus isolation and conventional RT-qPCR, have been reported [29]. Virus isolation is the gold standard for influenza virus diagnosis, but it is time-consuming, expensive, and not suitable for discrimination of multiple HPAI viruses. The conventional RT-qPCR assay is well-known and useful for molecular detection of influenza viruses such as influenza virus subtyping and positive detection using matrix gene detection [30]. In particular, this method detects the fluorescence signal of a positive sample in real time due to using of the target-specific probe/primers, resulting in high sensitivity and specificity. Therefore, the RT-qPCR assay could be widely used as molecular diagnostic technology in clinical fields. To detect H5 HPAI virus and difference of H5 HPAI clade viruses in clinical samples, a multiplex RT-qPCR assay has been developed using several pairs of primers and probes $[29,31,32]$. However, some drawbacks are the reduced specificity and sensitivity of the target gene amplification due to the nonspecific interaction among primers and probes in the same reaction [32].

In this study, novel primers and probes were designed for the pan-H5 HPAI detection and the classification of two clades of H5 HPAI, 2.3.4.4 or 2.3.3.1, in field samples. Our one-step multiplex RT-qPCR assay was successfully employed to amplify viruses and IAV-positive field samples using novel primers/probes for the simultaneous detection of pan-H5 HPAI and two clades without cross-reactivity. To enhance the specificity of target detection, we designed a new single primer set and each probe for the conserved sequences of H5 HPAI. After testing the IAV-positive clinical field samples, 51 were H5 HPAI positive and the cycle threshold values of these positive samples ranged from around 20 to 24 . Moreover, all H5 HPAI-positive samples were sequenced, and the results were verified by GenBank BLAST processing. Interestingly, the limit of detection of pan-H5 HPAI and clades in the singleplex and multiplex RT-qPCR assays was the same when our new single primer set and each probe combination was used.

\section{Conclusions}

Our one-step multiplex RT-qPCR method ensures highly specific and sensitive simultaneous detection of pan-H5 HPAI and two clades of H5 HPAI. Our method development focused on pan-H5 HPAI and two clades of H5 HPAI virus, 2.3.2.1 and 2.3.4.4, which have been dominant in Asia and present in Eurasia and Africa. This detection platform provides useful screening of clinical samples from domestic farms and live poultry markets.

Author Contributions: T.B.L., H.K.K. and W.N. performed experiments. V.P.L., D.S. and M.-S.S. analyzed the data. D.G.J. and S.-W.Y. designed the experiments and wrote the manuscript. All authors have read and agreed to the published version of the manuscript.

Funding: This research was supported by grants of the KRIBB Initiative program, supported by the BioNano Health-Guard Research Center funded by the Ministry of Science, ICT \& Future Planning (MSIP) of Korea as Global Frontier Project (Grant No. H-GUARD 2013M3A6B2078954), and supported by the Bio \& Medical Technology 
Development Program of the National Research Foundation (NRF) funded by the Ministry of Science \& ICT (NRF-2018M3A9H4056347)

Acknowledgments: We thank the following colleagues of Bon-Sang Koo (Korea Research Institute of Bioscience and Biotechnology, South Korea) excellent technical assistance in BL3 facility and also thank Sook-San Wang and Zanin Mark (Guangzhou Medical University, China) for informative discussions.

Conflicts of Interest: The authors declare no conflict of interest. The funders had no role in the design of the study; in the collection, analyses, or interpretation of data; in the writing of the manuscript, or in the decision to publish the results.

\section{References}

1. Yoon, S.W.; Webby, R.J.; Webster, R.G. Evolution and Ecology of Influenza A Viruses. Curr. Top. Microbiol. Immunol. 2014, 385, 359-375.

2. Tong, S.; Zhu, X.; Li, Y.; Shi, M.; Zhang, J.; Bourgeois, M.; Yang, H.; Chen, X.; Recuenco, S.; Gomez, J.; et al. New World Bats Harbor Diverse Influenza A Viruses. PLoS Pathog. 2013, 9, e1003657. [CrossRef] [PubMed]

3. Smith, G.J.; Donis, R.O. Nomenclature Updates Resulting from the Evolution of Avian Influenza A(H5) Virus Clades 2.1.3.2a, 2.2.1, and 2.3.4 During 2013-2014. Influenza Other Respir. Viruses 2015, 9, 271-276. [CrossRef] [PubMed]

4. Abolnik, C. Evolution of H5 Highly Pathogenic Avian Influenza: Sequence Data Indicate Stepwise Changes in the Cleavage Site. Arch. Virol. 2017, 162, 2219-2230. [CrossRef]

5. The WHO Report. Available online: http://www.who.int/influenza/human_animal_interface/H5N1_ cumulative_table_archives/en/) (accessed on 25 November 2019).

6. Sonnberg, S.; Webby, R.J.; Webster, R.G. Natural History of Highly Pathogenic Avian Influenza H5N1. Virus Res. 2013, 178, 63-77. [CrossRef] [PubMed]

7. de Vries, E.; Guo, H.; Dai, M.; Rottier, P.J.; van Kuppeveld, F.J.; de Haan, C.A. Rapid Emergence of Highly Pathogenic Avian Influenza Subtypes from a Subtype H5N1 Hemagglutinin Variant. Emerg. Infect. Dis. 2015, 21, 842-846. [CrossRef]

8. Kwon, J.H.; Lee, D.H.; Swayne, D.E.; Noh, J.Y.; Yuk, S.S.; Erdene-Ochir, T.O.; Hong, W.T.; Jeong, J.H.; Jeong, S.; Gwon, G.B.; et al. Reassortant Clade 2.3.4.4 Avian Influenza A(H5N6) Virus in a Wild Mandarin Duck, South Korea, 2016. Emerg. Infect. Dis. 2017, 23, 822-826. [CrossRef]

9. Gu, M.; Zhao, G.; Zhao, K.; Zhong, L.; Huang, J.; Wan, H.; Wang, X.; Liu, W.; Liu, H.; Peng, D.; et al. Novel Variants of Clade 2.3.4 Highly Pathogenic Avian Influenza A(H5N1) Viruses, China. Emerg. Infect. Dis. 2013, 19, 2021-2024. [CrossRef]

10. Kim, H.K.; Jeong, D.G.; Yoon, S.W. Recent Outbreaks of Highly Pathogenic Avian Influenza Viruses in South Korea. Clin. Exp. Vaccine Res. 2017, 6, 95-103. [CrossRef]

11. Verhagen, J.H.; Herfst, S.; Fouchier, R.A. Infectious Disease. How a Virus Travels the World. Science 2015, 347, 616-617. [CrossRef]

12. Bi, Y.; Chen, J.; Zhang, Z.; Li, M.; Cai, T.; Sharshov, K.; Susloparov, I.; Shestopalov, A.; Wong, G.; He, Y.; et al. Highly Pathogenic Avian Influenza H5N1 Clade 2.3.2.1c Virus in Migratory Birds, 2014-2015. Virol. Sin. 2016, 31, 300-305. [CrossRef]

13. Wu, H.; Peng, X.; Xu, L.; Jin, C.; Cheng, L.; Lu, X.; Xie, T.; Yao, H.; Wu, N. Novel Reassortant Influenza A(H5N8) Viruses in Domestic Ducks, Eastern China. Emerg. Infect. Dis. 2014, 20, 1315-1318. [CrossRef] [PubMed]

14. Adlhoch, C.; Brown, I.H.; Angelova, S.G.; Balint, A.; Bouwstra, R.; Buda, S.; Castrucci, M.R.; Dabrera, G.; Dan, A.; Grund, C.; et al. Highly Pathogenic Avian Influenza A(H5N8) Outbreaks: Protection and Management of Exposed People in Europe, 2014/15 and 2016. Eur. Surveill. 2016, 21, 30419. [CrossRef] [PubMed]

15. Stoute, S.; Chin, R.; Crossley, B.; Gabriel Senties-Cue, C.; Bickford, A.; Pantin-Jackwood, M.; Breitmeyer, R.; Jones, A.; Carnaccini, S.; Shivaprasad, H.L. Highly Pathogenic Eurasian H5N8 Avian Influenza Outbreaks in Two Commercial Poultry Flocks in California. Avian. Dis. 2016, 60, 688-693. [CrossRef] [PubMed]

16. Huang, Y.; Tang, H.; Duffy, S.; Hong, Y.; Norman, S.; Ghosh, M.; He, J.; Bose, M.; Henrickson, K.J.; Fan, J.; et al. Multiplex Assay for Simultaneously Typing and Subtyping Influenza Viruses by Use of an Electronic Microarray. J. Clin. Microbiol. 2009, 47, 390-396. [CrossRef] [PubMed] 
17. Imai, M.; Ninomiya, A.; Minekawa, H.; Notomi, T.; Ishizaki, T.; Tashiro, M.; Odagiri, T. Development of H5-RT-LAMP (Loop-Mediated Isothermal Amplification) System for Rapid Diagnosis of H5 Avian Influenza Virus Infection. Vaccine 2006, 24, 6679-6682. [CrossRef]

18. Michael, K.; Harder, T.C.; Mettenleiter, T.C.; Karger, A. Diagnosis and Strain Differentiation of Avian Influenza Viruses by Restriction Fragment Mass Analysis. J. Virol. Methods 2009, 158, 63-69. [CrossRef]

19. Tsukamoto, K.; Ashizawa, H.; Nakanishi, K.; Kaji, N.; Suzuki, K.; Okamatsu, M.; Yamaguchi, S.; Mase, M. Subtyping of Avian Influenza Viruses H1 to H15 on the Basis of Hemagglutinin Genes by PCR Assay and Molecular Determination of Pathogenic Potential. J. Clin. Microbiol. 2008, 46, 3048-3055. [CrossRef]

20. Naguib, M.M.; Graaf, A.; Fortin, A.; Luttermann, C.; Wernery, U.; Amarin, N.; Hussein, H.A.; Sultan, H.; Al Adhadh, B.; Hassan, M.K.; et al. Novel Real-Time PCR-Based Patho- and Phylotyping of Potentially Zoonotic Avian Influenza A Subtype H5 Viruses at Risk of Incursion into Europe in 2017. Eur. Surveill. 2017, 22, 30435. [CrossRef]

21. The WHO Manual on Animal Influenza Diagnosis and Surveillance. Available online: https://www.who.int/ csr/resources/publications/influenza/whocdscsrncs20025rev (accessed on 1 May 2002).

22. The WHO Report. Available online: https://www.who.int/influenza/gisrs_laboratory/H5CompleteTree.pdf? $\mathrm{ua}=1$ (accessed on 16 October 2015).

23. Hu, T.; Song, J.; Zhang, W.; Zhao, H.; Duan, B.; Liu, Q.; Zeng, W.; Qiu, W.; Chen, G.; Zhang, Y.; et al. Emergence of Novel Clade 2.3.4 Influenza A(H5N1) Virus Subgroups in Yunnan Province, China. Infect. Genet. Evol. 2015, 33, 95-100. [CrossRef]

24. Lee, Y.J.; Kang, H.M.; Lee, E.K.; Song, B.M.; Jeong, J.; Kwon, Y.K.; Kim, H.R.; Lee, K.J.; Hong, M.S.; Jang, I.; et al. Novel Reassortant Influenza A(H5N8) Viruses, South Korea, 2014. Emerg. Infect. Dis. 2014, 20, 1087-1089. [CrossRef] [PubMed]

25. Nguyen, D.T.; Jang, Y.; Nguyen, T.D.; Jones, J.; Shepard, S.S.; Yang, H.; Gerloff, N.; Fabrizio, T.; Nguyen, L.V.; Inui, K.; et al. Shifting Clade Distribution, Reassortment, and Emergence of New Subtypes of Highly Pathogenic Avian Influenza A(H5) Viruses Collected from Vietnamese Poultry from 2012 to 2015. J. Virol. 2017, 91, 1708-1716. [CrossRef] [PubMed]

26. Wong, F.Y.; Phommachanh, P.; Kalpravidh, W.; Chanthavisouk, C.; Gilbert, J.; Bingham, J.; Davies, K.R.; Cooke, J.; Eagles, D.; Phiphakhavong, S.; et al. Reassortant Highly Pathogenic Influenza A(H5N6) Virus in Laos. Emerg. Infect. Dis. 2015, 21, 511-516. [CrossRef] [PubMed]

27. Kapczynski, D.R.; Sylte, M.J.; Killian, M.L.; Torchetti, M.K.; Chrzastek, K.; Suarez, D.L. Protection of Commercial Turkeys Following Inactivated or Recombinant H5 Vaccine Application against the 2015U.S. H5N2 Clade 2.3.4.4 Highly Pathogenic Avian Influenza Virus. Vet. Immunol. Immunopathol. 2017, 191, 74-79. [CrossRef] [PubMed]

28. Yang, Y.; Li, S.; Wong, G.; Ma, S.; Xu, Z.; Zhao, X.; Li, H.; Xu, W.; Zheng, H.; Lin, J.; et al. Development of a Quadruple qRT-PCR Assay for Simultaneous Identification of Highly and Low Pathogenic H7N9 Avian Influenza Viruses and Characterization against Oseltamivir Resistance. BMC Infect. Dis. 2018, 18, 406. [CrossRef] [PubMed]

29. Payungporn, S.; Chutinimitkul, S.; Chaisingh, A.; Damrongwantanapokin, S.; Buranathai, C.; Amonsin, A.; Theamboonlers, A.; Poovorawan, Y. Single Step Multiplex Real-Time RT-PCR for H5N1 Influenza A Virus Detection. J. Virol. Methods 2006, 131, 143-147. [CrossRef]

30. Spackman, E.; Senne, D.A.; Myers, T.J.; Bulaga, L.L.; Garber, L.P.; Perdue, M.L.; Lohman, K.; Daum, L.T.; Suarez, D.L. Development of a Real-Time Reverse Transcriptase PCR Assay for Type A Influenza Virus and the Avian H5 and H7 Hemagglutinin Subtypes. J. Clin. Microbiol. 2002, 40, 3256-3260. [CrossRef]

31. Zhang, Z.; Liu, D.; Hu, J.; Sun, W.; Liu, K.; Li, J.; Xu, H.; Liu, J.; He, L.; Jiang, D.; et al. Multiplex One-Step Real-Time PCR Assay for Rapid Simultaneous Detection of Velogenic and Mesogenic Newcastle Disease Virus and H5-Subtype Avian Influenza Virus. Arch. Virol. 2019, 164, 1111-1119. [CrossRef]

32. Heine, H.G.; Foord, A.J.; Wang, J.; Valdeter, S.; Walker, S.; Morrissy, C.; Wong, F.Y.; Meehan, B. A Detection of highly pathogenic zoonotic influenza virus $\mathrm{H} 5 \mathrm{~N} 6$ by reverse-transcriptase quantitative polymerase chain reaction. Virol. J. 2015, 12, 18-21. [CrossRef]

(C) 2020 by the authors. Licensee MDPI, Basel, Switzerland. This article is an open access article distributed under the terms and conditions of the Creative Commons Attribution (CC BY) license (http://creativecommons.org/licenses/by/4.0/). 